\title{
EAl Endorsed Transactions

\section{Fractional Flow Reserve: Comparison between Invasive and Non-invasive Methods for Calculation of FFR}

\author{
S. Starcevic ${ }^{1,2, *}$, S. Djorovic ${ }^{1,2}$ and N. Filipovic ${ }^{1,2}$ \\ ${ }^{1}$ Faculty of Engineering, University of Kragujevac, Serbia; \\ ${ }^{2}$ BioIRC Research and Development Center of Bioengineering, Kragujevac, Serbia;
}

\begin{abstract}
The heart disease that affects millions of people worldwide is coronary artery disease. It is caused by a narrowing or blocking of the arteries due to plaque which restricts blood flow, and reduces the amount of oxygen to the heart. Angiogram as a tool, which represents an X-ray examination of the blood vessels in the heart, is traditional tool that aid physicians in the treatment of disease. The severity of blood flow blockages in the coronary arteries is indicated by a fractional flow reserve (FFR) and allows physicians to identify which specific lesion or lesions are responsible for patient ischemia. In this paper, the mathematical model for measuring FFR is derived, and compared with results that were obtained from simulations and angiographically based methods. This analytical model and simulations help to measure values of FFR, by non-invasive methods, only by using reconstructed geometries of coronary arteries with stenosis.
\end{abstract}

Keywords: fractional flow reserve, stenosis, lesion, angiography.

Received on 13 June 2017, accepted on 02 December 2017, published on 28 February 2018

Copyright () 2018 S. Starcevic et al., licensed to EAI. This is an open access article distributed under the terms of the Creative Commons Attribution licence (http://creativecommons.org/licenses/by/3.0/), which permits unlimited use, distribution and reproduction in any medium so long as the original work is properly cited.

doi: 10.4108/eai.28-2-2018.154146

\section{Introduction}

Fractional flow reserve (FFR) is very important index of coronary stenosis. It represents the ratio between the maximum achievable blood flow in a stenotic vessel (coronary artery), and the theoretical maximum flow in normal coronary artery.

FFR can be calculated as the ratio of two pressures; aortic pressure $(\mathrm{Pa})$ and distal coronary pressure $(\mathrm{Pd})$. Values of these two pressures are obtained during maximal hyperaemia.

Fractional flow reserve measurements are easily taken during routine coronary angiography. It can be done by using a pressure wire across the lesion of interest and inducing the state of maximum blood flow, which thereby allow to determine if the narrowing is tight enough to cause ischemia [1]. Pressure wire is used to calculate ratio between $\mathrm{Pd}$ and $\mathrm{Pa}$. This determined ratio represents the potential decrease in coronary flow distal to coronary artery.

\footnotetext{
*Corresponding author. Email: strahinja.straya@gmail.com
}

When coronary arteries are severe, they can limit myocardial blood flow, resulting in myocardial ischemia. The lesion is flow-limiting and severe if the narrowing of the luminal diameter is estimated to be $70 \%$ or greater [2].

Figure 1 represents scale of FFR values. An FFR measurement of 1.0 indicates an artery with normal blood flow. If FFR value is above 0.80 ischemia is very unlikely. The blood flow blockage caused by narrowing, which is responsible for a patient's ischemia is identified by the value of FFR below 0.75 .

Although angiographic assessment is often the only decision making modality that is available in clinical institutions, and the angiographically methods for the measurement of coronary flow and lesion dimension are well established, there is lack of fundamental theory that can determine the pressure drop $(\Delta p)$ and hence FFR. The objective that this study weighs to introduce a predictive model and to validate it. 


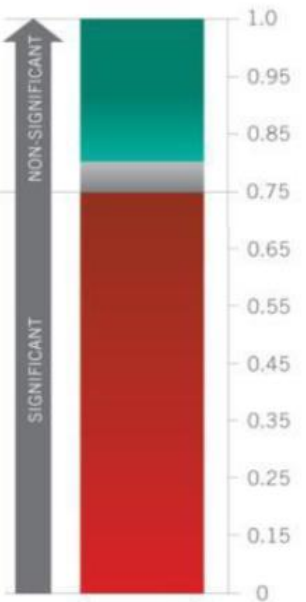

Figure 1. Scale of FFR values [1]

\section{Analytical model}

Fractional flow reserve represents functional parameter of stenosis severity. During hyperaemic flow FFR is expressed as:

$$
F F R=\frac{P_{\text {distal }}-P_{v}}{P_{a}-P_{v}}
$$

In Equation (1) $P_{a}$ is aortic pressure and it's equal to $P_{\text {proximal }} ; P_{v}$ is the central venous pressure; $P_{\text {proximal }}$ and $P_{\text {distal }}$ are the hyperaemic coronary pressures proximal and distal to stenosis, respectively [3].

Equation (1) can be approximated as:

$$
F F R=\frac{P_{\text {distal }}}{P_{a}}=\frac{P_{a}-\Delta p}{P_{a}}
$$

where $\Delta p$ is the pressure gradient along the axis of vessel segment from proximal to distal position of stenosis.

$$
\begin{aligned}
& \Delta P=\Delta P_{\text {convective }}+\Delta P_{\text {constriction }}+\Delta P_{\text {diffusive }} \\
& +\Delta P_{\text {expansion }}
\end{aligned}
$$

Equation (3) presents a model to determine $\Delta p$ for the coronary arteries. Gravity is negligible in the coronary circulation [4], the general Bernoulli equation can be written as:

$$
\Delta P_{\text {convective }}=\frac{\rho Q^{2}}{2}\left(\frac{1}{C S A_{\text {ouslet }}^{2}}-\frac{1}{C S A_{\text {inlet }}^{2}}\right)
$$

where Pconvective, Pconstriction, Pdiffusive and Pexpansion are energy losses due to flow convection, sudden constriction in cross-sectional areas from proximal normal vessel to stenosis, flow diffusion and sudden expansion in cross- sectional areas from stenosis to distal normal vessel, respectively [5].

$\mathrm{CSA}_{\text {inlet }}$ and $\mathrm{CSA}_{\text {outlet }}$ which are shown in equation (4) are the inlet and outlet cross-sectional areas, $Q$ is the hyperaemic flow rate in a vessel segment and is the density of blood.

When it happens that the flow transition is well-bound and follow the streamlines, energy losses due to sudden constriction is relatively small and negligible such that $\Delta \mathrm{P}_{\text {constriction }}=0$.

Viscosity in fully developed region is causing $P_{\text {diffusive }}$. Then the pressure drop serves both to accelerate the flow and to overcome viscous drag in the entrance region of stenosis which contributes that diffusive energy loss is developed [6].

From equation (5) the dimensionless radius of inviscid core $(\alpha)$ is developed for the entrance region of stenosis. If the $\alpha=r$ at the inlet and $0<\alpha<r$ from inlet to the fully developed region and $\alpha=0$ at the fully developed region then the flow velocity is uniform.

$$
\begin{aligned}
& \frac{\pi \mu L_{\text {stenosis }}}{4 \rho Q}= \\
& =\frac{1}{4} \int_{\alpha}^{1} \frac{(1-\alpha)(6+\alpha)\left(1+4 \alpha+9 \alpha^{2}+4 \alpha^{3}\right)}{5 \alpha(3+2 \alpha)\left(3+2 \alpha+\alpha^{2}\right)^{2}} d \alpha,
\end{aligned}
$$

where $L_{\text {stenosis }}$ is the length of stenosis [7]. $L_{\text {vessel }}$ is the length of vessel, which is composed of both normal vessel and stenosis.

Effects derived on entrance plus the viscosity leads to the diffusive loss of energy. In effort to determine value for FFR equation (3) is combined with equation (2).

\section{Results and discussion}

In this study, geometries of two left coronary arteries were reconstructed. There were two methods for reconstruction of these arteries. One of arteries was reconstructed from the computer tomography footage, and the other one was reconstructed by angiography footage.

When it comes to reconstruction from images of angiogram, it is important to choose coinciding frames in the same phase of the cardiac cycle. Chosen projections used for reconstruction are shown in figure 2.
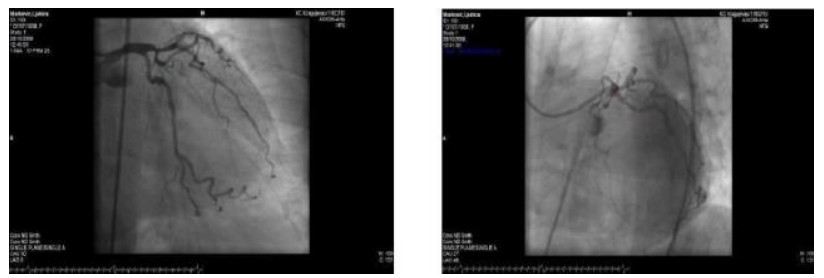

Figure 2. Images from angiogram 
For merging constructed central lines and outlines of these two projections, it is important to match characteristic points on each projection. When lines and points got merged the 3D model is derived.

For 3D model of second patient the computed tomography images (CT) were used. In the figure 3, which represents a frame taken from DICOM file, can be seen which artery has lesion. Using programs Mimics, Geomagic studio, 3D model of left coronary artery was made. Mimics is used for manipulation of footages of CT of certain patient. Images were imported in Mimics, where after manipulation 3D geometry is calculated. Calculated model is after imported as STL.file, in Geomagic studio. Next step is to generate finite element mesh and to prepare model for further analyses.

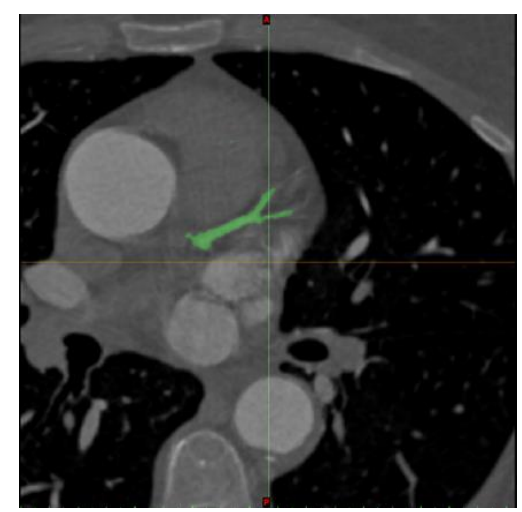

Figure 3. CT image of left coronary artery

After preparing 3D geometries of these two arteries the simulations and measurements for analytical model were made. This is done so the results will be compared with results that doctors obtained during angiography or with other invasive methods.

Figure 4 and figure 5 will represent pressure distribution through both reconstructed geometries of arteries, after simulations were done.

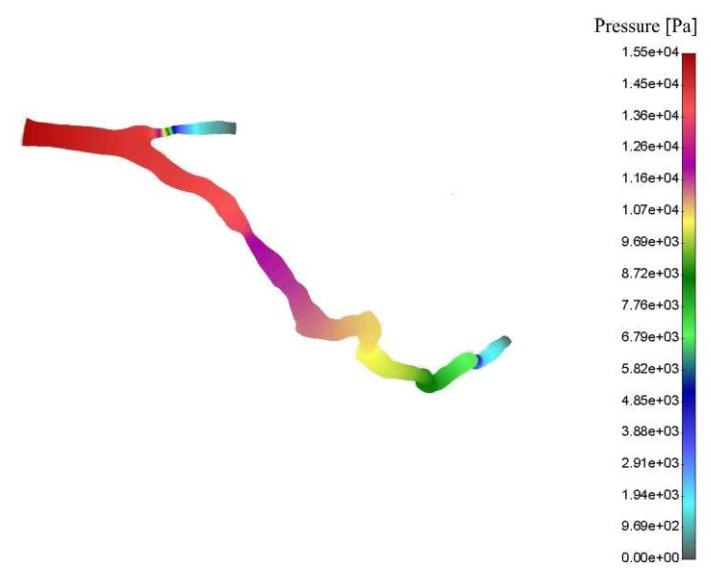

Figure 4. Pressure distribution of first patient $[\mathrm{Pa}]$

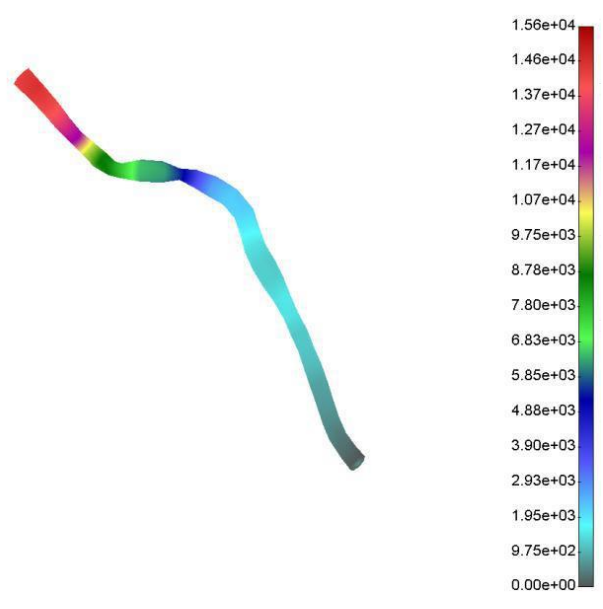

Figure 5. Pressure distribution of second patient $[\mathrm{Pa}]$

Values for FFR were obtained with three methods. First method is one which results are compared with results obtained from other two methods. Second method is based on obtaining dimensions from models' geometries, and used them in presented analytical model. Third method were simulations which gave pressure distribution, so it was possible to take values of pressures distal and proximal to stenosis and using them to derive value of FFR.

Compared results are presented in table 1:

Table 1. Values of FFR

\begin{tabular}{llll} 
Method & Angiography/CT & $\begin{array}{l}\text { Analytical } \\
\text { model }\end{array}$ & Simulation \\
\hline Patient 1 & 0.88 & 0.9141 & 0.92 \\
\hline Patient 2 & 0.71 & 0.7234 & 0.75
\end{tabular}

\section{Conclusion}

This study was validating analytical model and reliability of simulations for calculation of FFR values. As it was presented, analytical model was derived from General Bernoulli equation with diffusive energy loss, and due to a sudden expansion post-stenosis.

Those two methods were validated for two models of coronary arteries. For series of arteries analytical model needs to be modified, but simulation methods can work properly, no matter how many branches are on model.

The analytical model is very fast after getting a geometry compared with method use for simulations. Both of methods can be complementary for the noninvasive predictions of FFR.

This study will be extended, in the future, for larger group of patients. The main goal is that this model is widely used, but there are a lot of improvements for this 
analytical model. This model will be validated also with simulations, but is still concept which still develops.

\section{References}

[1] Shamla S., Bluestone, M.: Fractional Flow Reserve (FFR) \& FAME Family Studies. St. Jude Medical, Inc. Global Headquarters.

Available: [ http://www.sjm.com/ffr-fact-sheet]

[2] Huo, Y., Svendsen M., Choy, J.S., Zhang, Z.D., Kassab, G.S. (2012) A Validated Predictive Model Of Coronary Fractional Flow Reserve. J. R. Soc. Interface 9(71): 13251338.

[3] Pijls, N.H., van Son, J.A., Kirkeeide, R.L., De Bruyne, B., Gould, K.L. (1993) Experimental Basis Of Determining Maximum Coronary, Myocardial, And Collateral Blood Flow By Pressure Measurements For Assessing Functional Stenosis Severity Before And After Percutaneous Transluminal Coronary Angioplasty. Circulation 87: 13541367.

[4] Nichols, W.W., O'Rourke, M.F. (2005) McDonald's Blood Flow In Arteries: Theoretical, Experimental And Clinical Principles, 5th ed. (Hodder Arnold, London, UK).

[5] Molloi, S., Ersahin, A., Tang, J., Hicks, J., Leung, C.Y. (1996) Quantification Of Volumetric Coronary Blood Flow With Dual-Energy Digital Subtraction Angiography. Circulation 93: 1919-1927.

[6] Hamilos, M., Peace, A., Kochiadakis, G., Skalidis, E., Ntalianis, A., De Bruyne, B., Vardas, P. (2010) Fractional Flow Reserve: And Indispensable Diagnostic Tool In The Cardiac Catheterization Laboratory. Hellenic J. Cardiol. 51(2): 133-141.

[7] Fargie, D., Martin, B.W. (1971) Developing Laminar Flow In A Pipe Of Circular Cross-Section. Proc. R. Soc. Lond. 321: 461-476. 